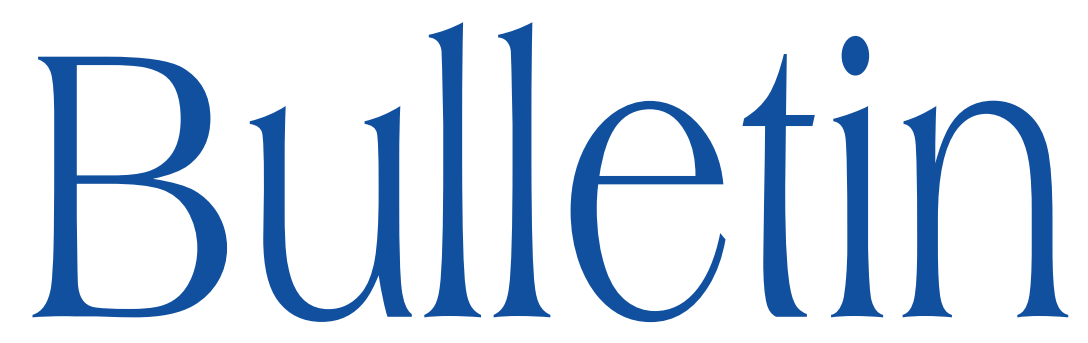

de la SOCIÉTÉ MATHÉMATIQUE DE FRANCE

\title{
WHEN IS A RIESZ DISTRIBUTION A COMPLEX MEASURE?
}

Alan D. Sokal

\section{Tome 139}

Fascicule 4

2011

\section{SOCIÉTÉ MATHÉMATIQUE DE FRANCE}

Publié avec le concours du Centre national de la recherche scientifique 


\title{
WHEN IS A RIESZ DISTRIBUTION A COMPLEX MEASURE?
}

\author{
By Alan D. Sokal
}

\begin{abstract}
Let $\mathscr{R}_{\alpha}$ be the Riesz distribution on a simple Euclidean Jordan algebra, parametrized by $\alpha \in \mathbb{C}$. I give an elementary proof of the necessary and sufficient condition for $\mathscr{R}_{\alpha}$ to be a locally finite complex measure (= complex Radon measure).

RÉSumé (Une distribution de Riesz, quand est-elle mesure complexe?)

Soit $\mathscr{R}_{\alpha}$ la distribution de Riesz sur une algèbre de Jordan euclidienne simple, paramétrisée par $\alpha \in \mathbb{C}$. Je donne une démonstration élémentaire de la condition nécessaire et suffisante pour que $\mathscr{R}_{\alpha}$ soit une mesure complexe localement finie (= mesure de Radon complexe).
\end{abstract}

\section{Introduction}

In the theory of harmonic analysis on Euclidean Jordan algebras (or equivalently on symmetric cones) [12], a central role is played by the Riesz distributions $\mathscr{R}_{\alpha}$, which are tempered distributions that depend analytically on a

Texte reçu le 16 juin 2009, révisé et accepté le 20 mai 2010.

Alan D. Sokal, Department of Physics, New York University, 4 Washington Place, New York, NY 10003 USA and Department of Mathematics, University College London, London WC1E 6BT, England • E-mail : sokal@nyu.edu

2000 Mathematics Subject Classification. - 43A85; 17A15, 17C99, 28C10, 44A10, 46F10, 47G10, 60E05, 62H05.

Key words and phrases. - Riesz distribution, Jordan algebra, symmetric cone, Gindikin's theorem, Wallach set, tempered distribution, positive measure, Radon measure, relatively invariant measure, Laplace transform. 
parameter $\alpha \in \mathbb{C}$. One important fact about the Riesz distributions is the necessary and sufficient condition for positivity, due to Gindikin [13]:

TheOREM 1.1. - [12, Theorem VII.3.1] Let $V$ be a simple Euclidean Jordan algebra of dimension $n$ and rank $r$, with $n=r+\frac{d}{2} r(r-1)$. Then the Riesz distribution $\mathcal{R}_{\alpha}$ on $V$ is a positive measure if and only if $\alpha=0, \frac{d}{2}, \ldots,(r-1) \frac{d}{2}$ or $\alpha>(r-1) \frac{d}{2}$.

The "if" part is fairly easy, but the "only if" part is reputed to be deep [13, 12 , 20]. (1)

The purpose of this note is to give a completely elementary proof of the "only if" part of Theorem 1.1, and indeed of the following strengthening:

THEOREM 1.2. - Let $V$ be a simple Euclidean Jordan algebra of dimension $n$ and rank $r$, with $n=r+\frac{d}{2} r(r-1)$. Then the Riesz distribution $\mathcal{R}_{\alpha}$ on $V$ is a locally finite complex measure [= complex Radon measure] if and only if $\alpha=0, \frac{d}{2}, \ldots,(r-1) \frac{d}{2}$ or $\operatorname{Re} \alpha>(r-1) \frac{d}{2}$.

This latter result is also essentially known [18, Lemma 3.3], but the proof given there requires some nontrivial group theory.

The idea of the proof of Theorem 1.2 is very simple: A distribution defined on an open subset $\Omega \subset \mathbb{R}^{n}$ by a function $f \in L_{\text {loc }}^{1}(\Omega)$ can be extended to all of $\mathbb{R}^{n}$ as a locally finite complex measure only if the function $f$ is locally integrable also at the boundary of $\Omega$ (Lemma 2.1); furthermore, this fact survives analytic continuation in a parameter (Proposition 2.3). In the case of the Riesz distribution $\mathscr{R}_{\alpha}$, a simple computation using its Laplace transform (Lemma 3.4) plus a bit of extra work (Lemma 3.5) allows us to determine the allowed set of $\alpha$, thereby proving Theorem 1.2.

Theorem 1.2 thus states a necessary and sufficient condition for $\mathscr{R}_{\alpha}$ to be a distribution of order 0 . It would be interesting, more generally, to determine the order of the Riesz distribution $\mathscr{R}_{\alpha}$ for each $\alpha \in \mathbb{C}$.

It would also be interesting to know whether this approach is powerful enough to handle the multiparameter Riesz distributions $\mathscr{R}_{\boldsymbol{\alpha}}$ with $\boldsymbol{\alpha}=\left(\alpha_{1}, \ldots, \alpha_{r}\right) \in \mathbb{C}^{r}[12$, Theorem VII.3.2] and/or the Riesz distributions on homogeneous cones that are not symmetric (i.e. not self-dual) and hence do not arise from a Euclidean Jordan algebra [13, 20].

In an Appendix I comment on a beautiful but little-known elementary proof of Theorem 1.1 - which does not extend, however, to Theorem 1.2 - due to Shanbhag [27] and Casalis and Letac [9].

(1) The set of values of $\alpha$ described in Theorem 1.1 is the so-called Wallach set [29, 30, 21, $10,11,12]$.

TOME $139-2011-\mathrm{N}^{\mathrm{O}} 4$ 


\section{A general theorem on distributions}

We assume a basic familiarity with the theory of distributions [26, 19] and recall some key notations and facts.

For each open set $\Omega \subseteq \mathbb{R}^{n}$, we define the space $\mathscr{D}(\Omega)$ of $C^{\infty}$ functions having compact support in $\Omega$, the corresponding space $\mathscr{D}^{\prime}(\Omega)$ of distributions, and the space $\mathscr{D}^{\prime k}(\Omega)$ of distributions of order $\leq k$. In particular, the space $\mathscr{D}^{\prime 0}(\Omega)$ consists of the distributions that are given locally (i.e. on every compact subset of $\Omega$ ) by a finite complex measure.

Let $f: \Omega \rightarrow \mathbb{C}$ be a measurable function, and extend it to all of $\mathbb{R}^{n}$ by setting $f \equiv 0$ outside $\Omega$. We say that $f \in L_{\text {loc }}^{1}(\Omega)$ if, for every $x \in \Omega, f$ is (absolutely) integrable on some neighborhood of $x$. Any $f \in L_{\text {loc }}^{1}(\Omega)$ defines a distribution $T_{f} \in \mathscr{D}^{\prime 0}(\Omega)$ by

$$
T_{f}(\varphi)=\int \varphi(x) f(x) d x \quad \text { for all } \varphi \in \mathscr{D}(\Omega)
$$

We are interested in knowing under what circumstances the distribution $T_{f} \in$ $\mathscr{D}^{\prime 0}(\Omega)$ can be extended to a distribution $\widetilde{T}_{f} \in \mathscr{D}^{\prime 0}\left(\mathbb{R}^{n}\right)$, i.e. one that is locally everywhere on $\mathbb{R}^{n}$ a finite complex measure.

Lemma 2.1. - Let $f: \Omega \rightarrow \mathbb{C}$ be in $L_{\mathrm{loc}}^{1}(\Omega)$, and let $T_{f} \in \mathscr{D}^{\prime 0}(\Omega)$ be the corresponding distribution. Then the following are equivalent:

(a) $f \in L_{\text {loc }}^{1}(\bar{\Omega})$, i.e. for every $x \in \bar{\Omega}, f$ is integrable on some neighborhood of $x .^{(2)}$

(b) There exists a distribution $\widetilde{T}_{f} \in \mathscr{D}^{\prime 0}\left(\mathbb{R}^{n}\right)$ that extends $T_{f}$ and is supported on $\bar{\Omega}$

(c) There exists a distribution $\widetilde{T}_{f} \in \mathscr{D}^{\prime 0}\left(\mathbb{R}^{n}\right)$ that extends $T_{f}$.

Proof. - (a) $\Longrightarrow(\mathrm{b})$ : It suffices to define $\widetilde{T}_{f}(\varphi)=\int_{\Omega} \varphi(x) f(x) d x$ for all $\varphi \in \mathscr{D}\left(\mathbb{R}^{n}\right)$.

(b) $\Longrightarrow$ (c) is trivial.

(c) $\Longrightarrow$ (a): By hypothesis, for every $x \in \partial \Omega$ and every compact neighborhood $K \ni x$, there exists a finite complex measure $\mu_{K}$ supported on $K$ such that $\widetilde{T}_{f}(\varphi)=\int \varphi d \mu_{K}$ for every $\varphi \in \mathscr{D}\left(\mathbb{R}^{n}\right)$ with support in $K$. But since $\widetilde{T}_{f}$ extends $T_{f}$, the restriction of $\mu_{K}$ to every compact subset of $K \cap \Omega$ must coincide with the measure $f(x) d x$. Since $K \cap \Omega$ is $\sigma$-compact, this implies that $\int|f(x)| d x=\left|\mu_{K}\right|(K \cap \Omega)<\infty$, so that $f$ is integrable in a neighborhood $K \cap \Omega$ of $x$.

(2) Since this has already been assumed for $x \in \Omega$, the content of hypothesis (a) is that it should hold also for $x \in \partial \Omega$. 
We now extend this idea to allow for analytic dependence on a parameter. Let $\Omega$ be an open set in $\mathbb{R}^{n}$, let $D$ be a connected open set in $\mathbb{C}^{m}$, and let $F: \Omega \times D \rightarrow \mathbb{C}$ be a continuous function such that $F(x, \cdot)$ is analytic on $D$ for each $x \in \Omega$. Then, for each $\lambda \in D$, define

$$
T_{\lambda}(\varphi)=\int \varphi(x) F(x, \lambda) d x \quad \text { for all } \varphi \in \mathscr{D}(\Omega) .
$$

Lemma 2.2. - With $F$ as above, the map $\lambda \mapsto T_{\lambda}$ is analytic from $D$ into $\mathscr{D}^{\prime}(\Omega)$ in the sense that $\lambda \mapsto T_{\lambda}(\varphi)$ is analytic for all $\varphi \in \mathscr{D}(\Omega)$.

Proof. - This is an immediate consequence of the hypotheses on $F$ together with standard facts about scalar-valued analytic functions in $\mathbb{C}$ (either Morera's theorem or the Cauchy integral formula) and $\mathbb{C}^{m}$ (e.g. the weak form of Hartogs' theorem).

Remark. - Weak analyticity in the sense used here is actually equivalent to strong analyticity: see e.g. [15, pp. 37-39, Théorème 1 and Remarque 1] [5, Theorems 3.1 and 3.2] [14, Theorem 1]. Indeed, our hypothesis on $F$ is equivalent to the even stronger statement that the map $\lambda \mapsto F(\cdot, \lambda)$ is analytic from $D$ into the space $C^{0}(\Omega)$ of continuous functions on $\Omega$, equipped with the topology of uniform convergence on compact subsets [15, p. 41, example (a)]. But we do not need any of these facts; weak analyticity is enough for our purposes.

Putting together these two lemmas, we obtain:

Proposition 2.3. - Let $F$ be as above, let $D_{0} \subseteq D$ be a nonempty open set, and let $\lambda \mapsto \widetilde{T}_{\lambda}$ be a (weakly) analytic map of $D$ into $\mathscr{D}^{\prime}\left(\mathbb{R}^{n}\right)$ such that $\widetilde{T}_{\lambda}$ extends $T_{\lambda}$ for each $\lambda \in D_{0}$. Then, for each $\lambda \in D$, we have:

(a) $\widetilde{T}_{\lambda}$ extends $T_{\lambda}$.

(b) If $\widetilde{T}_{\lambda} \in \mathscr{D}^{\prime 0}\left(\mathbb{R}^{n}\right)$, then $F(\cdot, \lambda) \in L_{\text {loc }}^{1}(\bar{\Omega})$.

Proof. - (a) This is immediate by analytic continuation: for each $\varphi \in \mathscr{D}(\Omega)$, both $\widetilde{T}_{\lambda}(\varphi)$ and $T_{\lambda}(\varphi)$ are (by hypothesis and Lemma 2.2, respectively) analytic functions of $\lambda$ on $D$ that coincide on $D_{0}$, therefore they must coincide on all of $D$.

(b) This is immediate from (a) together with Lemma 2.1.

We shall apply this setup with $F(x, \lambda)=f(x)^{\lambda}$ where $f: \Omega \rightarrow(0, \infty)$ is a continuous function; in fact, we shall take $f$ to be a polynomial.

TOME $139-2011-\mathrm{N}^{\mathrm{O}} 4$ 
Remark. - Let $P$ be a polynomial that is strictly positive on $\Omega$ and vanishes on $\partial \Omega$, and define for $\operatorname{Re} \lambda>0$ a tempered distribution $\mathscr{P}_{\Omega}^{\lambda} \in \phi^{\prime}\left(\mathbb{R}^{n}\right)$ by the formula

$$
\mathscr{P}_{\Omega}^{\lambda}(\varphi)=\int_{\Omega} P(x)^{\lambda} \varphi(x) d x \quad \text { for } \varphi \in \phi\left(\mathbb{R}^{n}\right)
$$

Then $\mathscr{P}_{\Omega}^{\lambda}$ is a tempered-distribution-valued analytic function of $\lambda$ on the right half-plane, and it is a deep result of Atiyah, Bernstein and S.I. Gelfand [3, $1,2,4]$ that $\mathscr{P}_{\Omega}^{\lambda}$ can be analytically continued to the whole complex plane as a meromorphic function of $\lambda$ with poles on a finite number of arithmetic progressions. It is important to note that our Proposition 2.3 does not rely on this deep result; rather, it says that whenever such an analytic continuation exists (however it may be constructed), the analytically-continued distribution $\mathscr{P}_{\Omega}^{\lambda}$ can be a complex measure only if $P^{\lambda} \in L_{\text {loc }}^{1}(\bar{\Omega})$.

\section{Application to Riesz distributions}

We refer to the book of Faraut and Korányi [12] for basic facts about symmetric cones and Jordan algebras. Let $V$ be a simple Euclidean (real) Jordan algebra of dimension $n$ and rank $r$, with Peirce subspaces $V_{i j}$ of dimension $d$; recall that $n=r+\frac{d}{2} r(r-1)$. We denote by $(x \mid y)=\operatorname{tr}(x y)$ the inner product on $V$, where $\operatorname{tr}$ is the Jordan trace and $x y$ is the Jordan product. Let $\Omega \subset V$ be the positive cone (i.e. the interior of the set of squares in $V$, or equivalently the set of invertible squares in $V$ ); it is self-dual, i.e. $\Omega^{*}=\Omega$. We denote by $\Delta(x)=\operatorname{det}(x)$ the Jordan determinant on $V$ : it is a homogeneous polynomial of degree $r$ on $V$, which is strictly positive on $\Omega$ and vanishes on $\partial \Omega$, and which satisfies [12, Proposition III.4.3]

$$
\Delta(g x)=\operatorname{Det}(g)^{r / n} \Delta(x) \quad \text { for } g \in G, x \in V,
$$

where $G$ denotes the identity component of the linear automorphism group of $\Omega$ [it is a subgroup of $G L(V)$ ] and Det denotes the determinant of an endomorphism. We then have the following fundamental Laplace-transform formula:

Theorem 3.1. - [12, Corollary VII.1.3] For $y \in \Omega$ and $\operatorname{Re} \alpha>(r-1) \frac{d}{2}=$ $\frac{n}{r}-1$, we have

$$
\int_{\Omega} e^{-(x \mid y)} \Delta(x)^{\alpha-\frac{n}{r}} d x=\Gamma_{\Omega}(\alpha) \Delta(y)^{-\alpha}
$$


where

$$
\Gamma_{\Omega}(\alpha)=(2 \pi)^{(n-r) / 2} \prod_{j=0}^{r-1} \Gamma\left(\alpha-j \frac{d}{2}\right) .
$$

Thus, for $\operatorname{Re} \alpha>(r-1) \frac{d}{2}$, the function $\Delta(x)^{\alpha-\frac{n}{r}} / \Gamma_{\Omega}(\alpha)$ is locally integrable on $\bar{\Omega}$ and polynomially bounded, and so defines a tempered distribution $\mathscr{R}_{\alpha}$ on $V$ by the usual formula

$$
\mathscr{R}_{\alpha}(\varphi)=\frac{1}{\Gamma_{\Omega}(\alpha)} \int_{\Omega} \varphi(x) \Delta(x)^{\alpha-\frac{n}{r}} d x \quad \text { for } \varphi \in \phi(V) .
$$

Using (5), a beautiful argument — which is a special case of Bernstein's general method for analytically continuing distributions of the form $\mathscr{P}_{\Omega}^{\lambda}[2,4]$ - shows that the Riesz distributions $\mathcal{R}_{\alpha}$ can be analytically continued to the whole complex $\alpha$-plane:

Theorem 3.2. - [12, Theorem VII.2.2 et seq.] The distributions $\mathcal{R}_{\alpha}$ can be analytically continued to the whole complex $\alpha$-plane as a tempered-distributionvalued entire function of $\alpha$. Furthermore, the distributions $\mathcal{R}_{\alpha}$ have the following properties:

$$
\begin{aligned}
\mathscr{R}_{0} & =\delta \\
\mathscr{R}_{\alpha} * \mathcal{R}_{\beta} & =\mathscr{R}_{\alpha+\beta} \\
\Delta(\partial / \partial x) \mathscr{R}_{\alpha} & =\mathscr{R}_{\alpha-1} \\
\Delta(x) \mathscr{R}_{\alpha} & =\left(\prod_{j=0}^{r-1}\left(\alpha-j \frac{d}{2}\right)\right) \mathcal{R}_{\alpha+1}
\end{aligned}
$$

(here $\delta$ denotes the Dirac measure at 0) and

$$
\mathscr{R}_{\alpha}\left(\varphi \circ g^{-1}\right)=\operatorname{Det}(g)^{\alpha r / n} \mathscr{R}_{\alpha}(\varphi) \quad \text { for } g \in G, \varphi \in \varnothing(V)
$$

(in particular, $\mathcal{R}_{\alpha}$ is homogeneous of degree $\alpha r-n$ ). Finally, the Laplace transform of $\mathscr{R}_{\alpha}$ is

$$
\left(\mathscr{L} \mathcal{R}_{\alpha}\right)(y)=\Delta(y)^{-\alpha}
$$

for $y$ in the complex tube $\Omega+i V$.

The property (8d) is not explicitly stated in [12], but for $\operatorname{Re} \alpha>(r-1) \frac{d}{2}$ it is an immediate consequence of (6)/(7), and then for other values of $\alpha$ it follows by analytic continuation (see also [18, Proposition 3.1(iii) and Remark 3.2]). Likewise, the property (9) is not explicitly stated in [12], but for $\operatorname{Re} \alpha>(r-1) \frac{d}{2}$ it is an immediate consequence of $(4) /(7)$, and then for other values of $\alpha$ it follows by analytic continuation (see also [18, Proposition 3.1(i)]). It follows 
from $(8 \mathrm{a}, \mathrm{b})$ that the distributions $\mathcal{R}_{\alpha}$ are all nonzero; and it follows from this and (9) that $\mathscr{R}_{\alpha} \neq \mathscr{R}_{\beta}$ whenever $\alpha \neq \beta$.

It is fairly easy to find a sufficient condition for the Riesz distributions to be a positive measure:

Proposition 3.3 ([12, Proposition VII.2.3], see also [18, Section 3.2], [21, 6])

(a) For $\alpha=k \frac{d}{2}$ with $k=0,1, \ldots, r-1$, the Riesz distribution $\mathscr{R}_{\alpha}$ is a positive measure that is supported on the set of elements of $\bar{\Omega}$ of rank exactly $k$ (which is a subset of $\partial \Omega$ ).

(b) For $\alpha>(r-1) \frac{d}{2}$, the Riesz distribution $\mathcal{R}_{\alpha}$ is a positive measure that is supported on $\Omega$ and given there by a density (with respect to Lebesgue measure) that lies in $L_{\mathrm{loc}}^{1}(\bar{\Omega})$.

The interesting and nontrivial fact (Theorem 1.1 above) is that the converse of Proposition 3.3 is also true: the foregoing values of $\alpha$ are the only ones for which $\mathcal{R}_{\alpha}$ is a positive measure. Here I shall use Proposition 2.3 together with the Laplace-transform formula $(5) /(10)$ to provide an alternate and extremely elementary proof of the stronger converse result stated in Theorem 1.2.

LEMMA 3.4. - $\Delta^{\lambda} \in L_{\mathrm{loc}}^{1}(\bar{\Omega})$ if and only if $\operatorname{Re} \lambda>-1$; or in other words, $\Delta^{\alpha-\frac{n}{r}} \in L_{\mathrm{loc}}^{1}(\bar{\Omega})$ if and only if $\operatorname{Re} \alpha>(r-1) \frac{d}{2}=\frac{n}{r}-1$.

Proof. - Since $|\Delta(x)|^{\lambda}=\Delta(x)^{\operatorname{Re} \lambda}$, it suffices to consider real values of $\lambda$.

For $\lambda>-1$ [i.e. $\alpha>(r-1) \frac{d}{2}$ ], fix any $y \in \Omega$ : the fact that the integral (5) is convergent, together with the fact that $x \mapsto e^{+(x \mid y)}$ is locally bounded, implies that $\Delta^{\lambda} \in L_{\text {loc }}^{1}(\bar{\Omega})$.

Now consider $\lambda=-1$ : again fix any $y \in \Omega$, and let $\mu=\inf _{x \in \bar{C}}(x \mid y)>0$ $\|x\|=1$

where $\|\cdot\|$ is any norm on $V$. Choose $R>0$ such that $|\Delta(x)| \leq 1$ whenever $\|x\| \leq R$. Then

$$
\int_{\substack{x \in \Omega \\\|x\| \leq R}} e^{-(x \mid y)} \Delta(x)^{-1} d x=\lim _{\lambda \downarrow-1} \int_{\substack{x \in \Omega \\\|x\| \leq R}} e^{-(x \mid y)} \Delta(x)^{\lambda} d x
$$

by the monotone convergence theorem. We now proceed to obtain a lower bound on

$$
M_{\lambda}:=\int_{\substack{x \in \Omega \\\|x\| \leq R}} e^{-(x \mid y)} \Delta(x)^{\lambda} d x .
$$


For any $\beta \geq 1$, we have

$$
\int_{\substack{x \in \Omega \\ \leq\|x\| \leq \beta R}} e^{-(x \mid y)} \Delta(x)^{\lambda} d x=\beta^{n+r \lambda} \int_{\substack{x \in \Omega \\ \frac{R}{2} \leq\|x\| \leq R}} e^{-\beta(x \mid y)} \Delta(x)^{\lambda} d x
$$

$\frac{\beta}{2} R \leq\|x\| \leq \beta R$

$$
\begin{aligned}
& \leq \beta^{n+r \lambda} e^{-(\beta-1) \frac{R}{2} \mu} \int_{x \in \Omega} e^{-(x \mid y)} \Delta(x)^{\lambda} d x \\
& \frac{R}{2} \leq\|x\| \leq R
\end{aligned}
$$

$$
\leq \beta^{n+r \lambda} e^{-(\beta-1) \frac{R}{2} \mu} M_{\lambda}
$$

where the first equality used the homogeneity of $\Delta$. Now sum this over $\beta=2^{k}$ $(k=1,2,3, \ldots)$; the sum is convergent, and we conclude that

$$
\int_{x \in \Omega} e^{-(x \mid y)} \Delta(x)^{\lambda} d x \leq C M_{\lambda}
$$

for a universal constant $C<\infty$ that is independent of $\lambda$ for $-1<\lambda \leq 0$. Since (5) tells us that

$$
\lim _{\lambda \downarrow-1} \int_{x \in \Omega} e^{-(x \mid y)} \Delta(x)^{\lambda} d x=+\infty
$$

due to the pole of the gamma function at $\alpha=(r-1) \frac{d}{2}$, we conclude that $\lim _{\lambda \downarrow-1} M_{\lambda}=+\infty$ as well. Therefore

$$
\int_{\substack{x \in \Omega \\\|x\| \leq R}} e^{-(x \mid y)} \Delta(x)^{-1} d x=+\infty,
$$

which proves that $\Delta^{-1} \notin L_{\text {loc }}^{1}(\bar{\Omega})$.

Since $\Delta$ is locally bounded, it also follows that $\Delta^{\lambda} \notin L_{\text {loc }}^{1}(\bar{\Omega})$ for $\lambda<-1$.

We shall also need a uniqueness result related to Proposition 3.3(a). If $\mu$ is a locally finite complex measure on $V$, we say that $\mu$ is $G$-relatively invariant with exponent $\kappa$ in case

$$
\mu(g A)=\operatorname{Det}(g)^{\kappa} \mu(A) \quad \text { for } g \in G, A \text { compact } \subseteq V .
$$

In particular, every such $\mu$ is $G \cap S L(V)$-invariant, i.e.

$$
\mu(g A)=\mu(A) \quad \text { for } g \in G \cap S L(V), A \text { compact } \subseteq V .
$$


Now define $\Omega_{k}=\{x \in \bar{\Omega}: \operatorname{rank}(x)=k\}$, so that $\partial \Omega=\bigcup_{k=0}^{r-1} \Omega_{k}$ and $\Omega=\Omega_{r}$. We then have the following result, which seems to be of some interest in its own right:

LEMmA 3.5. - (a) The group $G \cap S L(V)$ acts transitively on each set $\Omega_{k}$ $(0 \leq k \leq r-1)$.

(b) Let $\mu$ be a locally finite complex measure that is supported on $\Omega_{k}(0 \leq$ $k \leq r-1)$ and is $G \cap S L(V)$-invariant. Then $\mu$ is a multiple of $\mathcal{R}_{k d / 2}$.

(c) Let $\mu$ be a locally finite complex measure that is supported on $\partial \Omega$ and is $G$-relatively invariant with some exponent $\kappa$. Then there exists $k \in$ $\{0,1, \ldots, r-1\}$ such that $\mu$ is a multiple of $\mathcal{R}_{k d / 2}$ (and hence $\kappa=k d r / 2 n$ if $\mu \neq 0)$.

Proof. - (a) Fix a Jordan frame $c_{1}, \ldots, c_{r}$, and let $V=\bigoplus_{1 \leq i \leq j \leq r} V_{i j}$ be the corresponding orthogonal Peirce decomposition [12, Theorem IV.2.1]. Then, for $\lambda>0$, let $M_{\lambda}=P\left(c_{1}+\cdots+c_{r-1}+\lambda c_{r}\right) \in G L(V)$, where $P$ is the quadratic representation [12, p. 32]. From [12, p. 32 and Theorem IV.2.1(ii)] we see that $M_{\lambda}$ acts as multiplication by $\lambda^{2}$ on the space $V_{r r}$, as multiplication by $\lambda$ on the spaces $V_{i r}$ with $1 \leq i \leq r-1$, and as the identity on the other subspaces. ${ }^{(3)}$ We have $M_{\lambda} \in G$ [12, Proposition III.2.2] and $\operatorname{Det}\left(M_{\lambda}\right)=\lambda^{(r-1) d+2}=\lambda^{2 n / r}$.

Now write $e_{k}=c_{1}+\cdots+c_{k}$. By construction we have $M_{\lambda} e_{k}=e_{k}$ for $0 \leq k \leq r-1$. Now, we know [12, Proposition IV.3.1] that $\Omega_{k}=G e_{k}$, so that for any $x \in \Omega_{k}$ there exists $g \in G$ such that $x=g e_{k}$. Therefore, if we set $\lambda=\operatorname{Det}(g)^{-r / 2 n}$, we have $x=g M_{\lambda} e_{k}$ with $g M_{\lambda} \in G \cap S L(V)$.

(b) follows from (a) and Proposition 3.3(a) together with a standard result about the uniqueness of invariant measures: see e.g. [7, Chapitre 7, sec. 2.6, Théorème 3], [24, p. 138, Theorem 1] or [31, Theorem 7.4.1 and Corollary 7.4.2].

(c) is now an easy consequence, as we can write (uniquely) $\mu=\sum_{k=0}^{r-1} \mu_{k}$ with $\mu_{k}$ supported on $\Omega_{k}$, and each $\mu_{k}$ is $G$-relatively invariant with exponent $\kappa$ [since each set $\Omega_{k}$ is a separate $G$-orbit]. But in at most one case can $\kappa$ take the correct value $k d r / 2 n$; so all but one of the measures $\mu_{k}$ must be zero.

Remarks. - 1. Assertions (a) and (b) are false when $k=r$ : the determinant $\Delta(x)$ is invariant under the action of $G \cap S L(V)$ [cf. (4)], so $G \cap S L(V)$ cannot act transitively on $\Omega_{r}$; and all the measures $\mathcal{R}_{\alpha}$ with $\operatorname{Re} \alpha>(r-1) \frac{d}{2}$ are $G$-relatively invariant [hence $G \cap S L(V)$-invariant] and supported on $\Omega_{r}$.

2. A slight weakening of Lemma 3.5(b) - in which " $G \cap S L(V)$-invariant" is replaced by " $G$-relatively invariant with some exponent $\kappa$ " — is asserted in [21, p. 391, Remarque 3], but the proof given there is insufficient (if it were valid, it

(3) More generally, we see that $P\left(\sum \lambda_{i} c_{i}\right)$ acts as multiplication by $\lambda_{i} \lambda_{j}$ on $V_{i j}$. 
would apply also to $k=r$ ). However, Michel Lassalle has kindly communicated to me a simple alternative proof of this result, based on [21, Théorème 3 and Proposition 11(b)].

3. Further information on the Riesz measures $\mathcal{R}_{k d / 2}$ for $0 \leq k \leq r-1$ can be found in $[21,6]$.

Proof of Theorem 1.2. - We already know from Proposition 3.3(b) that $\mathcal{R}_{\alpha}$ is a locally finite complex measure for $\operatorname{Re} \alpha>(r-1) \frac{d}{2}$. On the other hand, by applying Proposition 2.3 to $F(x, \alpha)=\Delta(x)^{\alpha-\frac{n}{r}} / \Gamma_{\Omega}(\alpha)$ and using Lemma 3.4, we deduce that $\mathscr{R}_{\alpha}$ is not a locally finite complex measure whenever $\operatorname{Re} \alpha \leq$ $(r-1) \frac{d}{2}$ and $\Gamma_{\Omega}(\alpha) \neq \infty$. So it remains only to study the values of $\alpha$ for which $\Gamma_{\Omega}(\alpha)=\infty$, namely $\alpha \in\left\{0, \frac{d}{2}, \ldots,(r-1) \frac{d}{2}\right\}-\mathbb{N}$. For $\alpha \in\left\{0, \frac{d}{2}, \ldots,(r-\right.$ 1) $\left.\frac{d}{2}\right\}$ we know from Proposition 3.3(a) that $\mathcal{R}_{\alpha}$ is a positive measure. For $\alpha \in\left(\left\{0, \frac{d}{2}, \ldots,(r-1) \frac{d}{2}\right\}-\mathbb{N}\right) \backslash\left\{0, \frac{d}{2}, \ldots,(r-1) \frac{d}{2}\right\}$, we know from Proposition 3.3(a) and (8c) that $\mathcal{R}_{\alpha}$ is a distribution supported on $\partial \Omega$; and by (9) and Lemma 3.5(b) we conclude that it cannot be a locally finite complex measure (here we use the fact that $\mathscr{R}_{\alpha} \neq \mathscr{R}_{\beta}$ when $\alpha \neq \beta$ ).

Remark. - For $\operatorname{Re} \alpha<0$, an alternate proof that $\mathcal{R}_{\alpha}$ is not a complex measure can be based on the following fact, which is a special case of the $N=0$ case of [19, Theorem 7.4.3] (compare [19, Theorem 7.3.1]) but can also easily be proven by direct computation:

Lemma 3.6. - Let $\Omega$ be a proper open convex cone in a real vector space $V$, and let $\Omega^{*} \subset V^{*}$ be the open dual cone. Let $T \in \phi^{\prime}(V) \cap \mathscr{D}^{\prime 0}(V)$ be a tempered distribution of order 0 (i.e. a polynomially bounded complex measure) that is supported in $\bar{\Omega}$. Then the Laplace transform $\mathscr{L} T$ is analytic in the complex tube $\Omega^{*}+i V^{*}$ and is bounded in every set $K+\Omega^{*}+i V^{*}$ where $K$ is a compact subset of $\Omega^{*}$.

It then follows from (10) that $\mathscr{R}_{\alpha}$ cannot be a locally finite complex measure when $\operatorname{Re} \alpha<0$, because $\Delta(y)^{-\alpha}$ is unbounded at infinity. This argument handles (without the need for Lemma 3.5) the cases $d=1$ (real symmetric matrices) and $d=2$ (complex hermitian matrices) in Theorem 1.2.

\section{Appendix A}

\section{Remarks on an elementary proof of Theorem 1.1}

Casalis and Letac [9, Proposition 5.1] have given an elementary proof of Theorem 1.1 that deserves to be more widely known than it apparently is. ${ }^{(4)}$

(4) Science Citation Index shows only ten publications citing [9], and six of these have an author in common with [9].

TOME $139-2011-\mathrm{N}^{\mathrm{O}} 4$ 
They employ a method due to Shanbhag [27, p. 279, Remark 3] — who proved Theorem 1.1 for the cases of real symmetric and complex hermitian matrices which they abstract as a general "Shanbhag principle" [9, Proposition 3.1]. Here I would like to abstract their method even further, with the aim of revealing its utter simplicity and beauty.

Let $V$ be a finite-dimensional real vector space, and let $V^{*}$ be its dual space. We then make the following trivial observations:

(a) If $\mu$ is a positive (i.e. nonnegative) measure on $V$, then its Laplace transform

$$
\mathscr{L}(\mu)(y)=\int e^{-\langle y, x\rangle} d \mu(x)
$$

is nonnegative on any subset of $V^{*}$ where it is well-defined (i.e. where the integral is convergent).

(b) If $\mu$ is a positive measure on $V$, then so is $f \mu$ for every continuous (or even bounded measurable) function $f$ on $V$ that is nonnegative on $\operatorname{supp} \mu$.

(c) If $\mu$ is a (positive or signed) measure on $V$ whose Laplace transform is well-defined (and finite) on a nonempty open set $\Theta \subseteq V^{*}$, then the same is true for $P \mu$, where $P$ is any polynomial on $V$; furthermore, $\mathscr{L}(P \mu)=P(-\partial) \mathcal{L}(\mu)$. $^{(5)}$

Putting together these observations, we conclude:

\section{Proposition A.1 (Shanbhag-Casalis-Letac principle)}

If $\mu$ is a positive measure on $V$ whose Laplace transform is well-defined (and finite) on a nonempty open set $\Theta \subseteq V^{*}$, and $P$ is a polynomial on $V$ that is nonnegative on supp $\mu$, then $P(-\partial) \mathcal{L}(\mu) \geq 0$ everywhere on $\Theta$.

Remark. - Proposition A.1 also has a strong converse, which we shall state and prove at the end of this appendix.

Using Proposition A.1, we can give the following slightly simplified version of the Shanbhag-Casalis-Letac argument:

Proof of Theorem 1.1. - (Based on [9, Proposition 5.1].) In view of Proposition 3.3, it suffices to prove the converse statement. So let $\alpha \in \mathbb{R}$ and suppose that $\mathcal{R}_{\alpha}$ is a positive measure. Using Proposition A.1 with $P=\Delta$ together with the Laplace-transform formula (10), we conclude that

$$
\Delta(-\partial / \partial y) \Delta(y)^{-\alpha} \geq 0 \quad \text { for all } y \in \Omega .
$$

(5) Indeed, the same holds if the measure $\mu$ is replaced by a distribution $T \in \mathscr{D}^{\prime}(V)$. See [26, Chapitre VIII] or [19, Section 7.4] for the theory of the Laplace transform on $\mathscr{D}^{\prime}(V)$. 
But the "Cayley" identity [12, Proposition VII.1.4] tells us that

$$
\Delta(\partial / \partial y) \Delta(y)^{\lambda}=\Delta(y)^{\lambda-1} \prod_{j=0}^{r-1}\left(\lambda+j \frac{d}{2}\right),
$$

hence (since $\Delta$ is homogeneous of degree $r$ )

$$
\Delta(-\partial / \partial y) \Delta(y)^{-\alpha}=\Delta(y)^{-\alpha-1} \prod_{j=0}^{r-1}\left(\alpha-j \frac{d}{2}\right)
$$

It follows from (20) and (22) that $\mathscr{R}_{\alpha}$ is not a positive measure when $(r-2) \frac{d}{2}<$ $\alpha<(r-1) \frac{d}{2}$. But using the convolution equation (8b) with $\beta=d / 2$ together with the fact that $\mathcal{R}_{d / 2}$ is a positive measure [Proposition 3.3(a)], we conclude successively that $\mathscr{R}_{\alpha}$ is not a positive measure when $(k-1) \frac{d}{2}<\alpha<k \frac{d}{2}$ for any integer $k \leq r-1$. This leaves only negative multiples of $d / 2$; and the argument given after Lemma 3.6 shows that $\mathcal{R}_{\alpha}$ is not a positive measure whenever $\alpha<0$. $^{(6)}$

Remarks. - 1. This method has been used recently by Letac and Massam [22, proof of Proposition 2.3] to determine the set of acceptable powers $p$ for the noncentral Wishart distribution, generalizing the earlier proof of Shanbhag [27] and Casalis and Letac [9] for the ordinary Wishart distribution (which is essentially Theorem 1.1).

2. A very different proof of Theorem 1.1 for the cases $d=1,2$, using zonal polynomials, was given by Peddada and Richards [25, Theorems 1 and 3].

But this is not yet the end of the story; the proof can be further simplified. The use of the Laplace transform in the foregoing proof is in reality a red herring, as it is used twice in opposite directions: once in the proof of Proposition A.1, and once again in the proof of $(21) .{ }^{(7)}$ We can therefore give a direct proof that makes almost no reference to the Laplace transform:

Second proof of Theorem 1.1. - Consider first $(r-2) \frac{d}{2}<\alpha<(r-1) \frac{d}{2}$. If $\mathscr{R}_{\alpha}$ is a positive measure, then so is $\Delta(x) \mathscr{R}_{\alpha}$, which by (8d) equals $C_{\alpha} \mathscr{R}_{\alpha+1}$,

(6) Alternate argument: For $k=1,2,3, \ldots$ we know from Proposition $3.3(\mathrm{a}, \mathrm{b})$ and (9) that $\mathscr{R}_{k d / 2}$ is a positive measure that is not supported on a single point. If $\mathscr{R}_{-k d / 2}$ were a positive measure (recall that we know it is nonzero), then $\mathscr{R}_{k d / 2} * \mathscr{R}_{-k d / 2}$ could not be supported on a single point, contrary to the fact that $\mathscr{R}_{k d / 2} * \mathscr{R}_{-k d / 2}=\delta$ [cf. $\left.(8 \mathrm{a}, \mathrm{b})\right]$.

(7) The simplest proof of (21) is probably the one given in [12, Proposition VII.1.4], using Laplace transforms. However, direct combinatorial proofs are also possible: see [8] for a detailed discussion in the cases of real symmetric and complex hermitian matrices.

томе $139-2011-\mathrm{N}^{\mathrm{O}} 4$ 
where

$$
C_{\alpha}=\prod_{j=0}^{r-1}\left(\alpha-j \frac{d}{2}\right)<0 .
$$

It follows that $\mathscr{R}_{\alpha+1}$ must be a negative (i.e. nonpositive) measure. But this is surely not the case, as the Laplace-transform formula (10) immediately implies that no $\mathcal{R}_{\beta}$ can be a negative measure. ${ }^{(8)}$ This shows that $\mathcal{R}_{\alpha}$ is not a positive measure when $(r-2) \frac{d}{2}<\alpha<(r-1) \frac{d}{2}$. The proof is then completed as before. $^{(9)}$

It would be interesting to know whether this approach is powerful enough to handle the multiparameter Riesz distributions [12, Theorem VII.3.2] and/or the Riesz distributions on homogeneous cones that are not symmetric and hence do not arise from a Euclidean Jordan algebra [13, 20].

To conclude, let us give the promised strong converse to Proposition A.1:

Proposition A.2. - Let $T \in \mathscr{D}^{\prime}(V)$ be a distribution whose Laplace transform is well-defined on a nonempty open set $\Theta \subseteq V^{*}$. Let $S \subseteq V$ be a closed set, and suppose that there exists $y_{0} \in \Theta$ such that $[P(-\partial) \mathscr{L}(T)]\left(y_{0}\right) \geq 0$ for all polynomials $P$ on $V$ that are nonnegative on $S$. Then $T$ is in fact a positive measure that is supported on $S$.

Proof. - By replacing $T(x)$ by $e^{-\left\langle y_{0}, x\right\rangle} T(x)$, we can assume without loss of generality that $y_{0}=0$. Then the derivatives of $\mathscr{L}(T)$ at the origin give us the moments of $T$; and the hypothesis $[P(-\partial) \mathcal{L}(T)]\left(y_{0}\right) \geq 0$ implies, by Haviland's theorem [16, 17] [23, Theorem 3.1.2], that there exists a positive measure $\mu$ supported on $S$ that has these moments. Furthermore, the analyticity of $\mathscr{L}(T)$ in the open set $\Theta+i V^{*}$ implies that these moments satisfy a bound of the form $\left|c_{\mathbf{n}}\right| \leq A B^{|\mathbf{n}|} \mathbf{n}$ !, so that $\int e^{\epsilon|x|} d \mu(x)<\infty$ for some $\epsilon>0$. It follows that the Laplace transform $\mathscr{L}(\mu)$ is well-defined and analytic in a neighborhood of the origin; and since its derivatives at the origin agree with those of $\mathscr{L}(T)$, we must have $\mathscr{L}(\mu)=\mathscr{L}(T)$. But by the injectivity of the distributional Laplace transform [26, p. 306, Proposition 6], it follows that $\mu=T$.

(8) It would be interesting to know whether this residual use of the Laplace transform can be avoided. For $d \leq 2$ it can definitely be avoided, as $\alpha+1>(r-1) \frac{d}{2}$, so that $\mathscr{R}_{\alpha+1}$ is a nonzero positive measure by Proposition 3.3(b); but for $d>2$ I do not know.

(9) The argument given after Lemma 3.6 explicitly uses the Laplace transform. But the alternate argument given in footnote 6 does not. 
In Proposition A.2 it is essential that the Laplace transform of $T$ be welldefined on a nonempty open set $\Theta \ni y_{0}$, or in other words (when $y_{0}=0$ ) that $T$ have some exponential decay at infinity [in the sense that $\cosh (\epsilon|x|) T \in \phi^{\prime}(V)$ for some $\epsilon>0]$. It is not sufficient for $T$ to have finite moments of all orders satisfying $T(P) \geq 0$ for all polynomials $P$ on $V$ that are nonnegative on $S$. Indeed, Stieltjes' [28] famous example

$$
f(x)= \begin{cases}e^{-\log ^{2} x} \sin (2 \pi \log x) & \text { for } x>0 \\ 0 & \text { for } x \leq 0\end{cases}
$$

belongs to $\phi(\mathbb{R})$ and has zero moments of all orders [i.e. $T(P)=0$ for all polynomials $P$ ] but is not nonnegative.

\section{Acknowledgments}

I wish to thank Jacques Faraut for an extremely helpful conversation and correspondence, and in particular for pointing out a gap in my original proof of Theorem 1.2 (which used only Lemma 3.6 and hence was valid only for the cases $d=1,2)$. I also wish to thank Michel Lassalle for correspondence concerning Lemma 3.5, Muriel Casalis for correspondence and for providing me a copy of [6], and Christian Berg for correspondence concerning Proposition A.2.

I thank the Institut Henri Poincaré - Centre Emile Borel for hospitality during the programme on Interacting Particle Systems, Statistical Mechanics and Probability Theory (September-December 2008), where this work was almost completed.

This research was supported in part by U.S. National Science Foundation grant PHY-0424082.

\section{BIBLIOGRAPHY}

[1] M. F. Атіуан - "Resolution of singularities and division of distributions", Comm. Pure Appl. Math. 23 (1970), p. 145-150.

[2] I. N. BERNŠTEĬN - "Analytic continuation of generalized functions with respect to a parameter", Funkcional. Anal. i Priložen. 6 (1972), p. 26-40; English translation: Funct. Anal. Appl. 6 (1972), p. 273-285.

[3] I. N. BeRnŠteǏn \& S. I. GeL'FAND - "Meromorphy of the function $P^{\lambda}$ ", Funkcional. Anal. i Priložen. 3 (1969), p. 84-85; English translation: Funct. Anal. Appl. 3 (1969), p. 68-69.

[4] J.-E. BJÖRK - Rings of differential operators, North-Holland Mathematical Library, vol. 21, North-Holland Publishing Co., 1979. 
[5] J. Bochnak \& J. Siciak - "Analytic functions in topological vector spaces", Studia Math. 39 (1971), p. 77-112.

[6] M. Bonnefoy-CASAlis - "Familles exponentielles naturelles invariantes par un groupe", thèse de doctorat, Université Paul Sabatier de Toulouse, 1990.

[7] N. Bourbaki - Éléments de mathématique. Fascicule XXIX. Livre VI: Intégration. Chapitre 7: Mesure de Haar. Chapitre 8: Convolution et représentations, Actualités Scientifiques et Industrielles, No. 1306, Hermann, 1963.

[8] S. Caracciolo, A. Sportiello \& A. D. Sokal - "Combinatorial proofs of Cayley-type identities for derivatives of determinants and pfaffians", preprint arXiv:1105.6270.

[9] M. Casalis \& G. Letac - "Characterization of the Jørgensen set in generalized linear models", Test 3 (1994), p. 145-162.

[10] J. FARAUT - "Formule du binôme généralisée", in Harmonic analysis (Luxembourg, 1987), Lecture Notes in Math., vol. 1359, Springer, 1988, p. 170180.

[11] J. FARAUt \& A. KorÁNYI - "Function spaces and reproducing kernels on bounded symmetric domains", J. Funct. Anal. 88 (1990), p. 64-89.

[12] _ Analysis on symmetric cones, Oxford Mathematical Monographs, The Clarendon Press Oxford Univ. Press, 1994.

[13] S. G. Gindikin - "Invariant generalized functions in homogeneous domains", Funkcional. Anal. i Priložen. 9 (1975), p. 56-58; English translation: Funct. Anal. Appl. 9 (1975), p. 50-52.

[14] K.-G. Grosse-Erdmann - "A weak criterion for vector-valued holomorphy", Math. Proc. Cambridge Philos. Soc. 136 (2004), p. 399-411.

[15] A. Grothendieck - "Sur certains espaces de fonctions holomorphes. I", J. reine angew. Math. 192 (1953), p. 35-64.

[16] E. K. HaViland - "On the momentum problem for distribution functions in more than one dimension", Amer. J. Math. 57 (1935), p. 562-568.

[17] _ "On the momentum problem for distribution functions in more than one dimension. II", Amer. J. Math. 58 (1936), p. 164-168.

[18] J. Hilgert \& K.-H. Neeb - "Vector valued Riesz distributions on Euclidian Jordan algebras", J. Geom. Anal. 11 (2001), p. 43-75.

[19] L. HÖRMANDER - The analysis of linear partial differential operators. I, second ed., Springer Study Edition, Springer, 1990.

[20] H. IsHi - "Positive Riesz distributions on homogeneous cones", J. Math. Soc. Japan 52 (2000), p. 161-186.

[21] M. LASSAlle - "Algèbre de Jordan et ensemble de Wallach", Invent. Math. 89 (1987), p. 375-393. 
[22] G. Letac \& H. MAssam - "The noncentral Wishart as an exponential family, and its moments", J. Multivariate Anal. 99 (2008), p. 1393-1417.

[23] M. Marshall - Positive polynomials and sums of squares, Mathematical Surveys and Monographs, vol. 146, Amer. Math. Soc., 2008.

[24] L. NachBin - The Haar integral, D. Van Nostrand Co., Inc., Princeton, N.J.-Toronto-London, 1965.

[25] S. D. Peddada \& D. S. P. Richards - "Proof of a conjecture of M. L. Eaton on the characteristic function of the Wishart distribution", Ann. Probab. 19 (1991), p. 868-874; acknowledgment of priority 20 (1992), p. 1107.

[26] L. Schwartz - Théorie des distributions, Publications de l'Institut de Mathématique de l'Université de Strasbourg, vol. 9-10, Hermann, 1966.

[27] D. N. Shanbhag - "The Davidson-Kendall problem and related results on the structure of the Wishart distribution", Austral. J. Statist. 30A (1988), p. $272-280$.

[28] T. J. Stieltues - "Recherches sur les fractions continues", Ann. Fac. Sci. Toulouse 8 (1894), p. J1-J122, 9 (1895), p. A1-A47, reprinted, together with an English translation, in T. J. Stieltjes, Euvres complètes/Collected Papers, Springer, 1993, vol. II, p. 401-566 and 609-745.

[29] M. Vergne \& H. Rossi - "Analytic continuation of the holomorphic discrete series of a semi-simple Lie group", Acta Math. 136 (1976), p. 159 .

[30] N. R. WALlaCH - "The analytic continuation of the discrete series. I, II", Trans. Amer. Math. Soc. 251 (1979), p. 1-17, 19-37.

[31] R. A. WIJSMAN - Invariant measures on groups and their use in statistics, Institute of Mathematical Statistics Lecture Notes-Monograph Series, 14, Institute of Mathematical Statistics, 1990. 